\title{
Evaluating self-efficacy and personality differences of nursing students in clinical simulation
}

\author{
Constance E. McIntosh*1, Maria E. Hernandez-Finch ${ }^{2}$, Cynthia M. Thomas ${ }^{1}$, W. Holmes Finch ${ }^{2}$, Asia R. Hulse ${ }^{2}$, \\ Pamela K. Brelage ${ }^{1}$, David E. Mclntosh ${ }^{3}$ \\ ${ }^{1}$ School of Nursing, Ball State University, Muncie, Indiana, USA \\ ${ }^{2}$ Department of Educational Psychology, Ball State University, Muncie, Indiana, USA \\ ${ }^{3}$ Department of Special Education, Ball State University, Muncie, Indiana, USA
}

Received: February 8, 2020

DOI: $10.5430 /$ jnep.v10n9p12
Accepted: April 3, 2020

URL: https://doi.org/10.5430/jnep.v10n9p12

\begin{abstract}
Background: This present research was conducted to evaluate the efficacy of a clinical simulation where senior nursing students cared for a standardized patient with Autism Spectrum Disorder (ASD). The goal of the simulation was to teach the nursing students how to work with children with autism. In addition, the study aimed to determine if individual differences in personality affect students' abilities to complete the simulation and how a student's personality may affect their perceptions of the simulation. Projected outcomes included learning the use of appropriate communication strategies, improved assessment skills, prioritization of care, development of problem-solving skills, and decision-making abilities when dealing with children with ASD.

Methods: Simulations are verified as effective training mechanisms to increase students' self-efficacy in multiple nursing settings. Therefore, seventy-five senior baccalaureate nursing students completed the standardized patient simulation for care of an individual with ASD. The effect on the students' self-efficacy was measured using the Occupational and Academic Self-Efficacy for Nursing Measure, the IPEP-NEO short form, and an ASD simulation study questionnaire.

Results and conclusions: Logistic regression was used to investigate the relationship between personality measures and experience with ASD. The higher the openness and extraversion scores the more likely respondents were to disclose positive benefits in relation to expectations, communication strategies, teamwork, and reflection.
\end{abstract}

Key Words: Simulation, Autism spectrum disorder, Self-efficacy, Personality, Nursing education

\section{INTRODUCTION}

Nursing is a profession that has a minimal competency level conferred by a licensure exam (i.e., National Council Examination; NCLEX). Once licensed a registered nurse (RN) can work in a variety of settings including, but not limited to, the emergency department (ED), schools, hospital departments (e.g., maternal-child, medical-surgical, \& orthopedics), and community-based facilities (e.g., home health, hospice, \& clinics). As such, nurses must be able to demon- strate self-efficacy in any setting. Nursing simulations have been empirically supported as useful tools for helping students to develop clinical skills ${ }^{[1,2]}$ and have been shown to be effective for increasing students' self-efficacy in a variety of nursing roles. ${ }^{[3]}$ Simulations are learning opportunities where students can enact scenarios which arise in a variety of clinical settings. Therefore, nursing simulations may be useful for helping nurses thrive in the variety of settings they may encounter.

*Correspondence: Constance E. McIntosh; Email: cemcintosh@bsu.edu; Address: School of Nursing, Ball State University, Muncie, Indiana, USA. 
It is essential to expose nursing students to real life scenarios in preparation for transition to professional practice. With this is mind it is vital that students are prepared to deal with the challenges that may be presented when caring for a disabled client. The American Hospital Association ${ }^{[4]}$ indicates that $10 \%-25 \%$ of patients admitted to a hospital have a disability. One in 59 individuals are diagnosed with autism spectrum disorder (ASD) ${ }^{[5]}$ This warrants the inclusion of ASD educational content for students at all levels in a baccalaureate nursing program. Educators can better help students to understand the unique challenges and behaviors of individuals (i.e., patients) with ASD by assigning the students to care for these individuals during their clinical rotations. However, since it cannot be guaranteed that there will be a client with this diagnosis on the unit during a specific clinical rotation a simulation utilizing a standardized patient (SP) is an ideal way for students to gain experience and develop self-efficacy. Use of a standardized patient as opposed to a mannequin allows for development of communication skills which is vital when caring for an individual with a communication barrier as is commonly seen with ASD. In order for comfort to be developed there needs to be exposure. This led researchers to investigate ways to help nursing students feel more comfortable and effective when presented with challenging situations in various nursing specialties. Additionally, investigators were interested in discovering if a student's personality affected their expression of self-efficacy.

Twibell and colleagues ${ }^{[6]}$ define self-efficacy as including not only feelings of confidence but also how one feels about their performance ability to achieve positive outcomes. Zulkosky ${ }^{[7]}$ traces the definition of self-efficacy to Bandura's social learning theory where a person's ability is described in relation to feelings, thought, behavior, and motivation. Bandura $^{[8]}$ further states that a higher level of self-efficacy is developed by experiential mastery and repeated successes. Other work has defined self-efficacy as a person's belief about their ability to cope with a range of broad, pressing situations. ${ }^{[9]}$ Self-efficacy in samples of nursing students has been demonstrated to be positively associated with a variety of positive outcomes, including better evaluations of clinical performance, ${ }^{[10]}$ increased motivation to select and complete challenging tasks, ${ }^{[11]}$ and a greater willingness to view challenges as opportunities. ${ }^{[12]}$ In contrast, low selfefficacy in samples of nursing students has been shown to be associated with avoidance of tasks that are likely to result in failure, where students view challenges as obstacles that cannot be defeated. ${ }^{[10]}$ Thus, one way that simulations likelihood of increasing self-efficacy is by allowing students repeated opportunities to take on, and with practice, work through the difficult challenges and eventually have a level of competency, even excellence. Additionally, simulations provide a means for students to receive crucial, and immediate, feedback and support, which has also been shown to increase self-efficacy. ${ }^{[13]}$

There have been studies conducted investigating the role of various simulations on nursing students' self-efficacy ${ }^{[14,15]}$ and a literature review of self-efficacy of nursing students when using high-fidelity simulators. ${ }^{[16]}$ Further, a majority of the work published supports that the use of simulations results in increased self-efficacy of nursing students. ${ }^{[3]}$ For example, previous work by Franklin \& Lee ${ }^{[17]}$ found that simulation-based training was more effective compared to traditional lectures in increasing self-efficacy and providing nurses opportunities to obtain mastery in clinical skills. Other work has also maintained that simulations not only increase self-efficacy, but also increase self-confidence and result in student's providing better patient care. ${ }^{[18]}$ Importantly, nurses also perceive simulations as being effective for increasing their self-efficacy. ${ }^{[17]}$ Given these findings, it does not come as a surprise that many nursing programs have shifted their teaching practices for nursing students from classroom lectures to simulation laboratories. For example, one study found that $87 \%$ of 1,060 nursing programs regularly utilize nursing simulations. ${ }^{[19]}$ However, $81 \%$ of the 1,060 programs surveyed also reported a desire to utilize simulations more often. As such, research over the use of simulations is necessary to provide empirically supported, specific simulations that fit various scenarios.

The aforementioned research demonstrated the potential effects of simulations on students' self-efficacy. However, a select few researchers have also noted that individual difference factors may affect the effectiveness of simulations for some students. ${ }^{[3]}$ For example, research by Gosselin ${ }^{[20]}$ found that as students' tendencies to experience anxiety decreased, the effectiveness of nursing simulations increased. Other work has supported that personality dimensions, such as those posited in the Five-Factor Model explained by McCrae \& John, ${ }^{[21]}$ may impact the effectiveness of simulations by means of influencing decision making, memory, interpersonal functioning, and emotional reactance to difficult situations. ${ }^{[22]}$ Thus, this means that for some individuals, simulations may not be as effective. However, because little work has been done to investigate the impact of these characteristics on the effectiveness of nursing simulations, it is difficult to say with certainty which aspects of personality are most influential.

After a review of published nursing simulation research, no work was found that investigated the relationship between a 
simulation for working with individuals with ASD and personality or self-efficacy of nursing students. Key words for data base search included: personality keywords (combined with OR) of personality, personality trait, personality type and personality characteristic; search terms for CINAHL, Medline, and Psychinfo (combined with personality key terms) included nursing student, autism, autism spectrum, autism spectrum disorder, and disorders listed under this diagnosis (e.g., Rett's, Asperger's). Nurses report feeling hesitant about working with individuals with ASD this population and according to Rooth and Olinder ${ }^{[23]}$ have asked for additional education due to difficulty with communication and fear of behavioral issues that may be the result of stressful situations. Specifically, ASD is characterized by social and communication impairments and restrictive or repetitive behaviors. ${ }^{[24]}$ Social communication disturbances have various manifestations including an inability to maintain successful back and forth communication, inability to make friends, limited speech, narrow range of interests, inappropriate responses, or being completely non-verbal. Examples of restricted or repetitive behaviors that may be exhibited are problems with organization, need for rigid inflexible schedules, hypersensitivity to sounds or lights, and stimming, flapping, or rocking. Thus, as individuals with ASD present with varying symptoms and typically do not interact in a social setting with ease it is necessary for a nurse to be prepared to provide personalized care that addresses these difficulties. Simulations may allow for nurses to practice and become more comfortable in providing this care.

\section{Objectives}

The present research conducted a simulation that provided senior baccalaureate nursing students an opportunity to practice working with a standardized patient (SP) on the autism spectrum. Additionally, the study provided a means to gauge the effect of the simulation on student's self-efficacy when caring for a child with a disability. The goal of this work was to better prepare students to use appropriate communication strategies, effectively assess patients, and prioritize care for patients with ASD or cognitive/social impairments. The study also aimed to provide baccalaureate nursing students opportunities to employ appropriate problem-solving skills and make appropriate decisions as the simulation progressed.

To obtain a measure of self-efficacy the present study used The Occupational and Academic Self-Efficacy for Nursing Measure created by McLaughlin, Moutray, and Muldoon, ${ }^{[25]}$ adapted from the larger form of the Occupational Self-Efficacy Scale by Betz \& Hackett. ${ }^{[26]}$ Past research using the Occupational and Academic Self-Efficacy for Nursing Measure revealed that occupational self-efficacy predicted the achievement of academic success via grades. ${ }^{[25]}$ The results also supported that those students that set their own educational goals were empowered to be self-confident, committed, and motivated are more likely to be successful. ${ }^{[25]}$ Thus, the Occupational and Academic Self-Efficacy for Nursing Measure is useful for measuring changes in student's self-efficacy. However, the McLaughlin and colleagues ${ }^{[25]}$ study did not assess students' abilities to perform clinical duties or their confidence level after training. Instead, the authors only assessment of academic success was related to personality tests results and grades. As such, the present study aimed to also incorporate a means to address how simulations may affect clinical performance and confidence especially when students are caring for patients with ASD or other social/cognitive impairments.

\section{METHODS}

\subsection{Participants and setting}

Participants for this study were enrolled in a senior nursing leadership management course at a mid-size Midwestern University. All students voluntarily agreed to participate and were offered extra credit to do so. For those that chose not to participate, a different form of extra credit was provided. The primary investigator (PI) was not the course instructor and was not involved in any manner of teaching or grading the course. In addition, the primary course instructor and secondary course faculty (i.e., clinical faculty) were not present during the recruitment information session. A total of 75 students participated in the study, with 74 of them enrolled in a traditional BSN nursing degree. Ages ranged from 21 to $25(\mathrm{n}=73)$, with 4 males and 71 females. The majority of the students were Caucasian $(n=68)$. Most reported no prior experience with a patient with ASD (84\%). Most participants indicated never being required to repeat a nursing class ( $\mathrm{n}$ =64), while 9 participants indicated they have repeated one nursing class. Most participants have not had any prior experience with a patient with ASD $(n=63)$, but those who have had experience ranged from 1 day to 3 years and consisted of mostly child experience. Approximately one-third ( $\mathrm{n}=$ 28) of the participants indicated they have had interactions with a person with ASD in an educational setting, while 46 participants had not. Two-thirds $(n=47)$ of participants indicated they had personal experience with a person with ASD, while 27 had not.

Students were assigned a random participant number for the subsequent research project and data storage. All documents were de-identified for the study and consent forms were obtained from all participants and stored separately from the data. The study took place in the simulation labs at the University campus. 


\subsection{Measures}

A simulation was created in response to the need for nursing students to understand how to care for a patient with autism. Patients on the spectrum have unique behaviors that may challenge nurses and nursing students during the care process. Using an individual diagnosed with autism to serve as a $\mathrm{SP}$ is not permissible due to the possibility of invoking common behaviors (e.g., stemming behaviors, rocking, biting) that are often exhibited by an individual with ASD. These behaviors could cause harm to the individual but also to the nursing students attempting to provide care. In addition, individuals with ASD potentially would not be consistent with behaviors for each time the simulation was run. Therefore, the use of an SP with the knowledge to accurately role-play a patient on the spectrum was necessary. A twenty-fouryear-old male was selected to serve as the SP for the autism simulation because he was employed as a behavioral specialist for teenagers on the spectrum for several years. The simulation took place in an emergency department treatment room. The SP had fractured his tibia and is at the point of treatment where he is ready for discharge instruction and discharge from the unit. The SP engaged in behaviors unique to a patient with ASD in an ER setting. The student participants provided patient education, obtained pre-discharge vital signs, examined the patient's injured leg, communicated with the patient and the patient's mother, and discharged the patient to home.

Prior to beginning the simulation, learners completed the Autism Spectrum Disorder Simulation Consent (a consent form for gathering data from the simulation experience), the Autism Spectrum Disorder Simulation Media Consent (a media consent form for this simulation study), and an ASD Simulation Study Demographic Questionnaire (questions related to age, gender, race/ethnicity, and previous interactions with persons and patients diagnosed with ASD).

In the weeks prior to the simulation, participants completed the 120-item inventory to measure their big five personality domains including: Extraversion (energetic and assertive), Agreeableness (sympathetic, kind, and affectionate), Conscientiousness (organized, thorough, and planful), Neuroticism (tense, moody, and anxious) and Openness to Experience (wide interests, and being imaginative and insightful; i.e., NEO). Participants took this personality inventory because the researchers would like to see in what ways personality and temperament influence the ability to benefit from the nursing simulation. The IPIP (International Personality Item Pool) NEO short form is an extremely well-validated measure and common measure of the "big five" personality traits used extensively throughout all social science research including nursing and psychological sciences. ${ }^{[27]}$ This ques-

Published by Sciedu Press tionnaire is used for personality assessment of individuals in areas of neuroticism, extraversion, and openness to experience, agreeableness, and conscientiousness. The last two items, agreeableness and conscientiousness, were added after the original creation of the standard and therefore are not included in the NEO acronym. The short form, which is 120 questions, takes participants between 10-20 minutes to complete according to the test materials and was selected over the 300-question form to keep the total amount of time each participant must spend on testing to a minimum without sacrificing research integrity. More information about this popular measure and a link to the specific questions can be found here: http://www.personal.psu.edu/ j5j/IPIP/ (IPEP-NEO). ${ }^{[28]}$

In the weeks prior, participants also completed the McLaughlin et al. ${ }^{[25]}$ questionnaire which included items pertaining to personality and occupational and academic self-efficacy. There is also a commercially well validated, 34-item instrument available from Insight Assessment ${ }^{[28]}$ that can be found at http://www.insightassessment.com.

After the simulations, participants completed the ASD Simulation Study Questionnaire which included questions related to the ASD simulation, including if the participant had an opportunity to use critical thinking skills, communication strategies, and decision-making skills. In addition, questions related to the pre-briefing and debriefing were included.

\subsection{Data analysis}

Logistic regression with a cumulative logits link function was used to investigate the relationship between the 5 personality measures (Extraversion, Agreeableness, Conscientiousness, Neuroticism, and Openness), as well as prior experience working with patients with ASD (Yes/No), with each of the 15 survey items. This model was used in the analysis because the dependent variables consisted of ordinal responses (5 categories) to survey items, ranging from Strongly Disagree to Strongly Agree. The logistic regression model with the cumulative logits link was designed for exactly this type of data structure. Not Applicable (NA) was also an option on the survey but was not included in the analysis. The assumption of parallel lines was assessed and found to hold for each of the logistic regression models. The proportion of variance in the dependent variable accounted for by the 5 variable model was estimated using the Nagelkerke $\mathrm{R}^{2}$ statistic. Finally, given that a total of 15 regression models were fit to the data (one for each survey item), the Benjamini-Hochberg procedure ${ }^{[29]}$ was used to control the false discovery rate. Scale reliability was estimated using the greatest lower bound, which has been shown to be the optimal approach for assessing reliability for scales such as those used in this study. ${ }^{[30]}$ 


\section{RESUlts}

Prior to describing the results of the logistic regression analysis, a description of the sample results for the personality measure is first warranted. Table 1 includes the sample mean, median, range, standard deviation (SD), and reliability estimate of the big 5 personality traits.

Table 1. Descriptive statistics for the NEO big 5 personality traits

\begin{tabular}{llllll}
\hline NEO Trait & Mean & Median & Range & SD & Reliability \\
\hline Openness & 21.76 & 14 & $1-96$ & 22.67 & 0.81 \\
Conscientiousness & 76.45 & 79 & $13-99$ & 21.43 & 0.88 \\
Extraversion & 63.21 & 64 & $5-98$ & 21.19 & 0.83 \\
Agreeableness & 67.03 & 71 & $11-99$ & 21.09 & 0.75 \\
Neuroticism & 32.01 & 28 & $1-97$ & 22.62 & 0.88 \\
\hline
\end{tabular}

These results demonstrate that the scale scores were in the typical range, and that scale reliability was acceptable. Table 2 includes the response frequencies (percentages) for each of the ASD survey items. In general, participants were likely to agree or strongly agree with each of the items. Indeed, more than $90 \%$ of participants responded with agree or strongly agree to items $1,4,5,6,7,11$, and 12. Furthermore, items $2,9,13$, and 15 had $80 \%$ of respondents indicate agree or strongly agree. The only question for which a majority of respondents did not agree or strongly agree was item 14. In summary, study participants generally expressed high levels of agreement to the majority of items on the survey.

Table 2. Response Frequencies for the ASD Survey Items

\begin{tabular}{lllllll}
\hline Item & NA & Strongly Disagree & Disagree & Neutral & Agree & Strongly Agree \\
\hline ASD 1 & 0 & 0 & 0 & $1(1.4 \%)$ & $21(28.4 \%)$ & $52(70.3 \%)$ \\
ASD 2 & $3(4.0 \%)$ & $1(1.3 \%)$ & $2(2.7 \%)$ & $6(8.0 \%)$ & $30(40.0 \%)$ & $33(44.0 \%)$ \\
ASD 3 & $2(2.7 \%)$ & $1(1.3 \%)$ & $5(6.7 \%)$ & $15(20.0 \%)$ & $35(46.7 \%)$ & $17(22.7 \%)$ \\
ASD 4 & 0 & 0 & 0 & $3(4.0 \%)$ & $27(36.0 \%)$ & $45(60.0 \%)$ \\
ASD 5 & 0 & 0 & 0 & 0 & $22(29.3 \%)$ & $53(70.7 \%)$ \\
ASD 6 & 0 & 0 & 0 & 0 & $30(40.0 \%)$ & $45(60.0 \%)$ \\
ASD 7 & 0 & 0 & 0 & $1(1.3 \%)$ & $21(28.0 \%)$ & $53(70.7 \%)$ \\
ASD 8 & $1(1.3 \%)$ & $1(1.3 \%)$ & $4(4.0 \%)$ & $14(18.7 \%)$ & $28(37.3 \%)$ & $28(37.3 \%)$ \\
ASD 9 & $1(1.3 \%)$ & 0 & $4(5.3 \%)$ & $11(14.7 \%)$ & $36(48.0 \%)$ & $20(26.7 \%)$ \\
ASD 10 & 0 & $4(5.3 \%)$ & 0 & $1(1.3 \%)$ & $27(38.6 \%)$ & $39(55.7 \%)$ \\
ASD 11 & $2(2.9 \%)$ & $1(1.3 \%)$ & 0 & $3(4.0 \%)$ & $26(34.7 \%)$ & $46(61.3 \%)$ \\
ASD 12 & 0 & 0 & $4(5.3 \%)$ & $5(6.7 \%)$ & $36(48.0 \%)$ & $29(38.7 \%)$ \\
ASD 13 & 0 & $1(1.3 \%)$ & $24(32.0 \%)$ & $16(21.3 \%)$ & $17(22.7 \%)$ & $7(9.3 \%)$ \\
ASD 14 & $1(1.3 \%)$ & $10(13.3 \%)$ & 0 & $7(9.6 \%)$ & $36(49.3 \%)$ & $28(38.4 \%)$ \\
ASD 15 & 0 & $2(2.7 \%)$ & & $40.5 \%)$ & $32(43.2 \%)$ \\
\hline
\end{tabular}

With respect to the study participants, 73 of the 75 individuals were aged between 18 and 25 years, 71 of the 75 identifying as females, and 68 of the participants identified as white/non-Hispanic. Participants were asked several questions regarding their experience working with individuals identified with ASD. Results appear in Table 3. These results reveal that the majority $(84.0 \%)$ had no previous experience working with ASD individuals, and $86.6 \%$ had no coursework that touched on ASD. Of those who did report having worked with people with identified as ASD, nearly all cases involved children under the age of 17 years. Despite the relative lack of work experience with ASD, $62.7 \%$ of the participants did report some personal experience with individuals identified with ASD, and $28 \%$ reported interacting with ASD individuals in an educational setting.

The statistically significant results of the logistic regression model $(\alpha=0.05)$ appear in Table 4 . As a reminder, due to the large number of statistical tests that were conducted in this analysis, the Benjamini-Hochberg correction was used to control the Type I error rate. As the results in Table 4 
reveal, openness was found to be positively related to survey question 1 , with the model accounting for $32 \%$ of the variance in the item response. Thus, the higher the openness score, the more likely respondents were to agree that the ASD simulation provided a realistic example of what to expect when caring for a patient with ASD. Extraversion was positively associated with items 2,7 , and 11 . These results indicate that individuals with higher levels of extraversion were more likely to agree that they used communication strategies learned in class to communicate with ASD patients, that their group worked as a team during the simulation, and that the de-briefing provided them with an opportunity to reflect on their nursing interventions during the simulation. The logistic regression models accounted for $25 \%, 30 \%$, and $28 \%$ of the variance in items 2,7 , and 11 , respectively. Finally, item 1 was the only one to which prior experience with ASD individuals was related. In this case, those who did not have such experience were less likely to agree that the simulation provided a realistic example of what to expect when caring for a patient with ASD.

Table 3. Response frequencies to prior ASD experience items

\begin{tabular}{lll}
\hline Item & Response & $\begin{array}{l}\text { Frequency } \\
\text { (percent) }\end{array}$ \\
\hline ASD courses & 0 & $65(86.6 \%)$ \\
ASD experience & 1 & $9(12 \%)$ \\
Interaction in educational setting & Yes & $63(84 \%)$ \\
& No & $12(16 \%)$ \\
Personal experience with ASD & No & $28(37.3 \%)$ \\
& Yes & $46(61.3 \%)$ \\
& No & $27(36.7 \%)$ \\
\hline
\end{tabular}

Table 4. Logistic regression Coefficient Estimates, Standard Errors, Confidence Intervals, and Odds Ratios for Statistically Significant Results

\begin{tabular}{|c|c|c|c|c|}
\hline Predictor & Coefficient & Standard Error & Confidence Interval & Odds Ratio \\
\hline \multicolumn{5}{|c|}{ 1. ASD simulation provided a realistic example of what to expect when caring for patient with ASD. $R^{2}=0.32$} \\
\hline Openness & 0.04 & 0.02 & $(0.01,0.08)$ & 1.04 \\
\hline Prior experience & -2.37 & 0.88 & $(-4.08,-0.65)$ & 0.09 \\
\hline \multicolumn{5}{|c|}{ 2. I used communication strategies learned in class to communicate with the patient with ASD. $R^{2}=0.25$} \\
\hline Extraversion & 0.05 & 0.01 & $(0.02,0.07)$ & 1.05 \\
\hline \multicolumn{5}{|c|}{ 7. I believe my group worked as a team during the ASD simulation. $R^{2}=0.30$} \\
\hline Extraversion & 0.05 & 0.02 & $(0.02,0.08)$ & 1.05 \\
\hline \multicolumn{5}{|c|}{ 11. De-briefing provided an opportunity to reflect on my nursing interventions during the ASD simulation. $R^{2}=0.28$} \\
\hline Extraversion & 0.04 & 0.02 & $(0.01,0.08)$ & 1.04 \\
\hline
\end{tabular}

\section{Discussion}

Students were required to use clear, direct, and simple communication with an ASD SP and the patient's mother to complete a discharge assessment, provide discharge instructions for an ASD patient in an ED setting after experiencing an injury from home. The students would need to adjust their communication patterns based on the ASD SP's actions. Students were evaluated by faculty on their ability to initiate and complete assessment skills necessary for discharge depending on the SP's behaviors. Students were also evaluated on how they prioritized the care based on the behaviors exhibited by the SP and or the needs expressed by the mother which was also determined by the student's ability to problem-solve and make appropriate decisions. For example, the ASD SP would be non-verbal in some of the scenarios and verbal, using repetitive words or curse words in others. Students had to find alternative modes of communication in order to meet the needs of the patient. In some scenarios, the SP would not allow students to take his blood pressure or exhibited signs of increasing agitation so students would need to problem solve how to successfully get the blood pressure taken. All students in each simulation group were observed for their ability to work as a team and their contributions, or lack thereof, during the simulation.

In this study students were placed in small groups for the simulation experience in part to determine how the students functioned in as a group. It quickly became apparent to faculty some students failed to engage with the patient or the patient's mother and allowed other group members to take control. These students seemed uncomfortable with the SP's behaviors making it difficult to complete the necessary tasks. Other group members immediately assessed the situation, changed strategies if necessary, and quickly and confidently worked to provide appropriate care within the time frame 
provided. While many simulations are timed consideration should be given to not requiring a specific time frame for simulations where students are required to make judgement calls, and or when working with more challenging patients such as those with ASD or cognitive and social impairments. Allowing more time in a simulation may benefit those students with low openness and high neuroticism scores to formulate appropriate responses and become more comfortable in challenging situations.

When an individual understands his or her own personality type, they will be better able to address the behaviors and communication strategies of people with ASD or other disabilities. For example, a student scoring high in neuroticism may feel threatened when confronted by an ASD patient exhibiting physical and verbal behaviors. The student may be unable to critically think, make appropriate decisions, and problem solve to work though the challenging situation and provide the necessary care to the patient. Conversely, a student scoring low in neuroticism would tend to remain calm, controlled, and assess the situation making appropriate changes as necessary to provide care. ${ }^{[31]}$ These high and low neuroticism behaviors exhibited by some students were observed by faculty during the simulations.

\section{Conclusions}

It has been noted through this study that the higher the openness score, the more likely respondents were to agree that the ASD simulation provided a realistic example of what to expect when caring for a patient with ASD. Students scoring low in openness may not have had a previous experience with an ASD individual and may not have previewed the ASD video and information provided as part of the pre-briefing. In general, people scoring low on openness may not understand their own feelings in a situation, are less likely to express how they feel, and may not be as comfortable in unfamiliar situations. The pre-briefing in our study was therefore a necessary part of the simulation experience since the pre-briefing video and written information on ASD gave a realistic example of what behaviors an ASD person may exhibit in a healthcare environment.

Individuals with higher levels of extraversion were more likely to agree that they used communication strategies learned in class to communicate with patients with ASD, that their group worked as a team during the simulation, and that the de-briefing provided them an opportunity to reflect on their nursing interventions during the simulation. Faculty observed students who moved quickly once involved in the simulation and did not seem deterred from their tasks even when the SP became aggressive or passive (non-verbal and refusal to cooperate) but simply adapted to the changes. These students talked directly to the SP patient, inquired about favorite music or cars in an attempt to form a relationship with the SP.

It is essential to expose nursing students to real life healthcare situations via standardized patient simulations to allow introverted students more time to develop the communication techniques needed to work with this clientele. Nursing faculty have the ability to ensure that introverted students are as actively involved as extroverted students in simulation dynamics that allow them to take responsibility for patient care and interactions. Exposing students to the NEO exam or other personality trait predictors may offer insight into how a student may react to specific types of patients or situations when in a clinical environment. Simulations designed to place students in unpredictable situations and with challenging patient types permits students and faculty the opportunity to explore different strategies working with the individual students' personality traits to achieve positive outcomes. Simulation scenarios should also be designed to promote learning for people with different types of personality traits to be able to experience positive outcomes. In addition, it would be wise for nursing faculty to extend the debriefing process to provide adequate time for introverted students to formulate their thoughts and contribute valuable information. Introverted students may be less likely to engage in discussion when in groups of extroverted students. This does not mean the introverted students are less thoughtful, on the other hand, the introverted student may be more likely to think things through prior to reacting in a situation or discussion. Providing time allows, it may be wise to debrief in smaller groups of people or with individual students to allow more time for thoughtful reflection. Additionally, thoughtful reflection may come hours or days later and can be shared verbally or in writing, through the use of journaling, or using different modes of expression such as art, music, photography and film. It is important to remember the goal is to not change student's personalities but to help students identify and understand specific traits they exhibit and how these traits may be used to their benefit when caring for patients in a variety of healthcare environments.

\section{Limitations}

There were several limitations identified in this study. During this scenario, individual students interacted with the standardized patient. Future research is needed to address how a group or team interacts with an individual with ASD. This simulation was limited by scripted communication from the standardized patient. A scenario with non-structured communication would allow for additional applications of critical thinking skills. To further address the benefits to introverted 
students a comparison of immediate post simulation debriefing with one-week simulation debriefing for consistencies among personality types is warranted. This current study was limited to a one-time post simulation debriefing conducted with the same randomly assigned groups. There is the possibility a group dynamic between members might create a less than open environment when allowing for sharing feelings and performance. Controlled time frames for the simulation and the debriefing may also limit the thoughtful expression and reflection of more introverted students needing more time and alternative modes for self-reflection. Additionally, this study was limited by use of questionnaires and one standardized patient in the evaluation of personality types in relationship to care. Introducing case studies, videos, and toolkits that require students to consider their own feelings of openness or resistance to a person with ASD is needed in future research.

\section{CONFlicts OF INTEREST Disclosure}

The authors declare no conflict of interest.

\section{REFERENCES}

[1] Kaakinen J, Arwood E. Systematic review of nursing simulation literature for use of learning theory. International Journal of Nursing Scholarship. 2009; 6(1). https : //doi.org/10.2202/1548-923 $\mathrm{X} .1688$

[2] Raurell-Torredà M, Olivet-Pujol J, Romero-Collado À, et al. Casebased learning and simulation: Useful tools to enhance nurses' education?-Nonrandomized controlled trial. Journal of Nursing Scholarship. 2015; 47: 34-42. PMid:25346329 https ://doi .org/10.1 $111 / \mathrm{jnu} .12113$

[3] Lin H. Effectiveness of simulation-based learning on student nurses' self-efficacy and performance while learning fundamental nursing skills. Technology and Health Care. 2016; 24: 369-375. PMid:26444820 https : //doi.org/10.3233/THC-151094

[4] American Hospital Association. Fast facts on U.S. hospitals, 2019. [Internet] Available from: https://www.aha.org/statistics /fast-facts-us-hospitals

[5] Centers for Disease Control and Prevention. Autism prevalence slightly higher in CDC's ADDM network. 2018. [Internet] Available from: https://www.cdc.gov/media/releases/2018/p0 426-autism-prevalence.html

[6] Twibell R, Siela D, Riwitis C, et al. Nurses' perception of their self-confidence and the benefits and risks of family presence during resuscitation. American Journal of Critical Care. 2008; 17(2): 102112. PMid: 18310646 https://doi.org/10.4037/ajcc2008.1 7.2.101

[7] Zulkosky K. Self-efficacy: A concept analysis. Nursing Forum. 2009; 44(2): 93-102. https://doi .org/10.1111/j.1744-619 8.2009.00132.x

[8] Bandura A. Self-efficacy: Toward a unifying theory of behavioral change. Psychological Review. 1977; 84(2): 191-215. https : //doi.org/10.1037/0033-295X.84.2.191

[9] Luszczynska A, Scholz U, Schwarzer R. The general self-efficacy scale: Multicultural validation studies. The Journal of Psychology. 2005; 139(5): 439-457. PMid:16285214 https://doi.org/10.3 200/JRLP . 139 . 5.439-457

[10] Alavi NM. Self-efficacy in nursing students. Nursing and Midwifery Studies. 2014; 3(4): e25881. https ://doi.org/10.17795/nms journal25881

[11] Soudagar S, Rambod M, Beheshtipour N. Factors associated with nurses' self-efficacy in clinical setting in Iran. Iranian Journal of Nursing and Midwifery Research [Internet]. 2015; 20(2): 226-231.

[12] Zhang Z, Zhang C, Zhang X, et al. Relationship between self-efficacy beliefs and achievement in student nurses. Chinese Nursing Research.
2015; 2(2-3). https://doi.org/10.1016/j.cnre.2015.06.0 01

[13] Gibbons C. Stress, coping and burn-out in nursing students. International Journal of Nursing Studies. 2010; 47: 1299-309. PMid:20359710 https://doi.org/10.1016/j.ijnurstu. 201 0.02 .015

[14] Goldenberg D, Andrusyszyn M, Iwasiw C. The effect of classroom simulation on nursing students' self-efficacy related to health teaching. Journal of Nursing Education. 2005; 44(7): 310-314. https://doi.org/10.3928/01484834-20050701-04

[15] Kameg K, Howard V, Clockesy J, et al. The impact of high fidelity human simulation on self-efficacy of communication skills. Issues in Mental Health Nursing. 2010; 315-323. PMid:20394477 https://doi.org/10.3109/01612840903420331

[16] Leigh G. High-fidelity patient simulation and nursing students' selfefficacy: A review of the literature. International Journal of Nursing Education Scholarship. 2008; 5(1). PMid:18976234 https: //doi.org/10.2202/1548-923X.1613

[17] Franklin A, Lee C. Effectiveness of simulation for improvement in self-efficacy among novice nurses: A meta-analysis. Journal of Nursing Education. 2014; 53(11): 607-614. PMid:25350902 https ://doi.org/10.3928/01484834-20141023-03

[18] Brannan J, White A, Long J. Learning styles: Impact on knowledge and confidence in nursing students in simulation and classroom. International Journal of Nursing Education Scholarship. 2016; 13(1). PMid:27564700 https ://doi.org/10.1515/ijnes-2015-005 2

[19] National Council of State Boards of Nursing (NCSBN). [Internet] National simulation study: A longitudinal, randomized, controlled study replacing clinical hours with simulation in prelicensure nursing education. Journal of Nursing Regulation. 2014; 5(2): S3-S40. https : //doi .org/10.1016/S2155-8256(15)30062-4

[20] Gosselin A. Honor thesis and capstone: Nursing simulation experience self-efficacy, state, anxiety, locus of control and simulation effectiveness. University of New Hampshire: Scholar's Repository [Internet]. 2013; Available from: https://scholars.unh.edu/h onors/137/

[21] McCrae RR, John OP. An introduction to the five-factor model and its applications. Journal of Personality. 1992; 60(2): 175 215. PMid:1635039 https ://doi.org/10.1111/j.1467-6494. 1992.tb00970.x

[22] Weiler D, Gibson A, Saleem J. The effect of role assignment in high fidelity patient simulation on nursing students: An experimental research study. Nurse Education Today. 2018; 63: 29-34. 
PMid:29407257 https://doi.org/10.1016/j.nedt.2018.01 .012

[23] Rooth E, Olinder A. Nurses' experiences of giving care to children with autism spectrum disorder within somatic emergency care. Scientific Times Journal of Paediatrics. 2016; 1(1): 1-11.

[24] American Psychological Association. Diagnostic and statistical manual of mental disorders. 5th rev. ed. Washington, DC: Author; 2014.

[25] McLaughlin K, Moutray M, Muldoon OT. The role of personality and self-efficacy in the selection and retention of successful nursing students: A longitudinal study. Journal of Advanced Nursing 2008; 61(2): 211-221. PMid:18186912 https://doi.org/10.1111/j. 1365-2648.2007.04492. $\mathrm{x}$

[26] Betz N, Hackett G. Occupational Self-Efficacy Scale. 1998.

[27] Briley DA, Domiteaux M, Tucker-Drob EM. Achievement-relevant personality: Relations with the big five and validation of an efficient instrument. Learning and Individual Differences. 2014; 32: 2639. PMid:24839374 https ://doi .org/10.1016/j.lindif . 201 4.03 .010
[28] Insight Assessment. International Personality Item Pool: Representation of the BIO PI-R (IPIP-NEO). 2016. [Internet] Available from: http://www.personal.psu.edu/ j5j/IPIP/

[29] Benjamini Y, Hochberg Y. Controlling the false discovery rate: A practical and powerful approach to multiple testing. Journal of the Royal Statistical Society, Series B (methodological). 1995; 57(1): 289-300. https://doi.org/10.1111/j.2517-6161.19 95.tb02031.x

[30] Sijtsma K. On the use, the misuse, and the very limited usefulness of Cronbach's Alpha. Psychometrika. 2009; 74(1): 107-120. PMid:20037639 https://doi.org/10.1007/s11336-008-910 $1-0$

[31] Settles RE, Fischer S, Cyders MA, et al. Negative urgency: A personality predictor of externalizing behavior characterized by neuroticism, low conscientiousness, and disagreeableness. Journal of Abnormal Psychology. 2012; 121(1): 160-172. PMid:21859164 https://doi.org/10.1037/a0024948 